\section{Aquaporin 4 and glymphatic flow have central roles in brain fluid homeostasis}

\author{
Mootaz M. Salman (D), Philip Kitchen, Jeffrey J. Iliff and Roslyn M. Bill(D)
}

In their recent Review (MacAulay, $\mathrm{N}$. Molecular mechanisms of brain water transport. Nat. Rev. Neurosci. 22, 326-344 $(2021))^{1}$, MacAulay highlights many open questions about how brain water transport is controlled. They posit that cotransport of water may bridge the gap in our understanding of cellular and barrier brain water transport. As the existence of the glymphatic system and its dependence upon the glial water channel aquaporin 4 (AQP4) have been controversial, MacAulay places them outside the scope of their Review. We agree that a lack of mechanistic insight into them represents a significant gap in current knowledge of the brain in health and disease. However, it is necessary to contextualize the role of AQP4 in glymphatic function (which we think deserves more attention) and address the need for tighter definitions when describing the fluids involved. As MacAulay's review has such a broad title, our aim is to provide its reader with an appreciation of these important and, in some cases, emerging concepts in brain fluid dynamics.

As a detailed molecular mechanistic understanding of brain water transport is one indirectly. currently lacking, now is the time to carefully define the processes involved. The field has a tendency to discuss 'water' and 'fluid' in a manner that incorrectly suggests their interchangeability. As we describe in FIG. 1, in the glymphatic system, the clearance of brain waste occurs through paracellular flow. Classic tracer studies measure this paracellular flow, while the use of $\mathrm{H}_{2}{ }^{17} \mathrm{O}$ captures both paracellular flow and diffusive transcellular exchange of water ${ }^{2}$. Importantly, both are AQP4 dependent - one directly and

A comprehensive view of brain water transport must include the role of the glymphatic system (which refers to perivascular and paracellular fluid transport (FIG. 1)), especially in light of recent studies suggesting that perivascular cerebrospinal fluid (CSF) is a major source of oedema fluid that accumulates acutely following stroke ${ }^{3}$. The AQP4 dependence of perivascular flow is established: work in five independent laboratories ${ }^{4}$ has refuted the single study ${ }^{5}$ suggesting that this is an AQP4-independent process. The link between AQP4 and glymphatic function is compelling, not only from studies in $A q p 4^{-/-}$mice, but

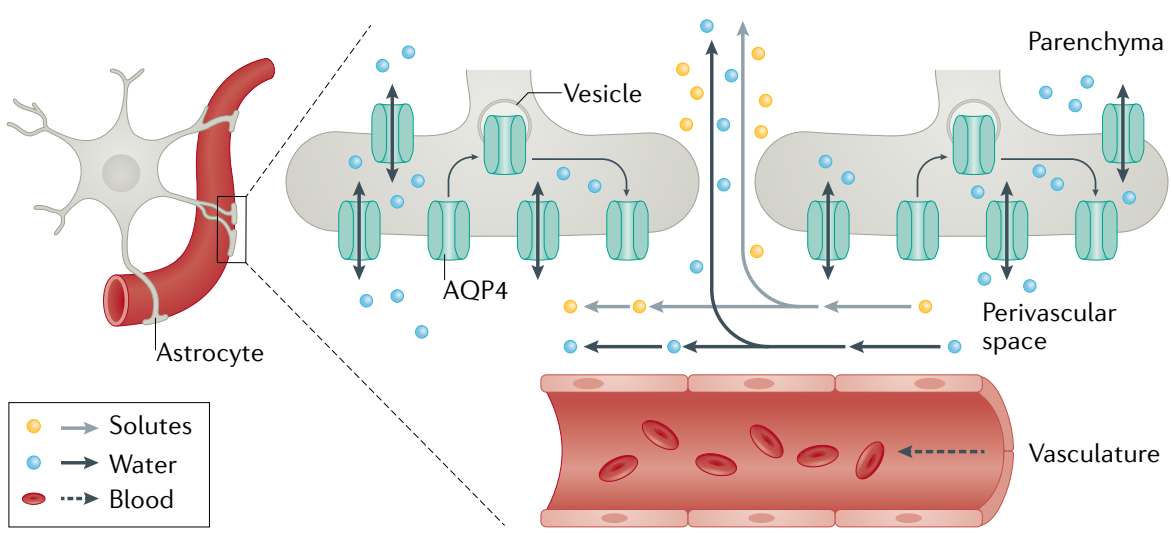

Fig. 1 | Aquaporin 4 has direct and indirect roles in controlling fluid flow in the brain. Astrocyte endfeet wrap around blood vessels at the blood-brain barrier, separated by a cerebrospinal fluid (CSF)-filled perivascular space. Para-arterial fluid influx, a trans-parenchymal pathway (where CSF exchanges with interstitial fluid to clear extracellular solutes) and para-venous efflux into the deep cervical lymph nodes define the glymphatic pathway. Glymphatic function clears brain metabolic waste. Solutes and water are transported paracellularly between astrocyte endfeet, while water can also be exchanged across the endfoot membrane via aquaporin 4 (AQP4). Physiological and pathophysiological factors regulate the relocalization of AQP4 between intracellular vesicular pools and the plasma membrane of astrocyte endfeet, dynamically regulating membrane water permeability. also Snta $^{-1-}$ and $m d x$ mice. Together, these studies confirm a requirement for polarized AQP4 localization for rapid tracer transport from the CSF into the brain parenchyma. We agree with MacAulay's view that a biophysical explanation for how AQP4 at astrocyte endfeet indirectly facilitates glymphatic flux is incompletely understood. However, we have shown that subcellular relocalization of AQP4, from intracellular vesicles to the plasma membrane, has a crucial role in the regulation of AQP4 function ${ }^{6}$ (FIG. 1). We $\mathrm{W}^{6}$ and others ${ }^{7,8}$ have also shown that AQP4 subcellular relocalization is a dynamic process independent of changes in AQP4 expression. Following pathological dysregulation, AQP4 relocalization to astrocyte endfeet facilitates oedema formation, which can be pharmacologically inhibited ${ }^{6}$. A comprehensive review of brain water transport should therefore consider the dynamic relocalization of AQP4 channels as it provides a framework to address fundamental questions about water homeostasis in health and disease.

One approach to answering some of the outstanding questions in the field is to use specific AQP4 inhibitors. Notably, it is here that much of the literature lacks clarity. TGN-020 has been suggested to be an AQP4 inhibitor on the basis of Xenopus laevis oocyte swelling assays, as have diverse, structurally unrelated molecules such as acetazolamide, ethoxzolamide, topiramate, lamotrigine, zonisamide, acetylsulfanilamide, phenytoin, bumetanide, furosemide, tetraethylammonium and IMD0354. The inhibitory action of the majority of these molecules has been challenged ${ }^{9}$ and many have AQP4-independent effects on brain water transport, confounding the interpretation of in vivo studies. To our knowledge, the off-target effects of TGN-020 remain completely unexplored. This highlights the need for well-validated inhibitors whose efficacies are reproducible between experimental assay systems and laboratories, and the need for greater understanding of the indirect effects of AQP4 knockout in the brain.

To conclude, we welcome MacAulay's Review $^{1}$; in combination with current knowledge of the glymphatic system, a new understanding for the role of dynamic AQP4 regulation and the search for specific inhibitors, understanding of the mechanisms of brain water transport can only improve.

There is a reply to this letter by MacAulay, N. Nat. Rev. Neurosci. https://doi.org/10.1038/ s41583-021-00515-y (2021).

Mootaz M. Salman (iD ${ }^{1,2 凶}$, Philip Kitchen ${ }^{3 凶}$, Jeffrey J. Iliff ${ }^{4,5,6 凶}$ and Roslyn M. Bill iD ${ }^{3 凶}$ 'Department of Physiology, Anatomy and Genetics, University of Oxford, Oxford, UK. 
${ }^{2}$ Oxford Parkinson's Disease Centre University of Oxford, Oxford, UK ${ }^{3}$ School of Biosciences, College of Health and Life Sciences, Aston University, Birmingham, UK

${ }^{4}$ Department of Psychiatry and Behavioral Sciences, University of Washington School of Medicine, Seattle, WA, USA

${ }^{5}$ Department of Neurology. University of Washington School of Medicine Seattle, WA, USA

${ }^{6}$ VISN 20 Mental IIIness Research, Education and Clinical Center, VA Puget Sound Health Care System, Seattle, WA, USA.

凶e-mail:mootaz.salman@dpag.ox.ac.uk; p.kitchen1@aston.ac.uk; jiliff@uw.edu. r.m.bill@aston.ac.uk

https://doi.org/10.1038/s41583-021-00514-z

1. MacAulay, N. Molecular mechanisms of brain water transport. Nat. Rev. Neurosci. 22, 326-344 (2021).

2. Alshuhri, M. S., Gallagher, L., Work, L. M. \& Holmes, W. M. Direct imaging of glymphatic transport using $\mathrm{H}_{2}{ }^{17} \mathrm{O}$ MRI. JCI Insight 6, e141159 (2021).

Mestre, $\mathrm{H}$. et al. Cerebrospinal fluid influx drives acute ischemic tissue swelling. Science 367 eaax7171 (2020).
4. Mestre, $\mathrm{H}$. et al. Aquaporin-4-dependent glymphatic solute transport in the rodent brain elife 7 , e40070 (2018).

5. Smith, A. J., Yao, X., Dix, J. A., Jin, B.-J. \& Verkman, A. S. Test of the 'glymphatic' hypothesis demonstrates diffusive and aquaporin-4-independent solute transport in rodent brain parenchyma. eLife 6, e27679 (2017).

6. Kitchen, P. et al. Targeting aquaporin-4 subcellular localization to treat central nervous system edema. Cell 181, 784-799 (2020).

7. Ciappelloni, S. et al. Aquaporin-4 surface trafficking regulates astrocytic process motility and synaptic activity in health and autoimmune disease. Cell Rep. 27, 3860-3872 (2019)

8. Lisjak, M., Potokar, M., Rituper, B., Jorgačevski, J. $\&$ Zorec, R. AQP4e-based orthogonal arrays regulate rapid cell volume changes in astrocytes. J. Neurosci. 37, 10748-10756 (2017).

9. Yang, B., Zhang, H. \& Verkman, A. S. Lack of aquaporin-4 water transport inhibition by antiepileptics and arylsulfonamides. Bioorg. Med. Chem. 16, 7489-7493 (2008).

Competing interests

The authors declare no competing interests.

\section{Peer review information}

Nature Reviews Neuroscience thanks R. Keep and the other, anonymous, reviewer(s) for their contribution to the peer review of this work.

\section{Reply to 'Aquaporin 4 and glymphatic flow have central roles in brain fluid homeostasis'}

\section{Nanna MacAulay (D)}

I thank Mootaz Salman, Philip Kitchen, Jeffrey Iliff and Roslyn Bill for their additions to my recent Review (Molecular mechanisms of brain water transport. Nat. Rev. Neurosci. 22, 326-344 (2021)) $)^{1}$. In their correspondence, the authors argue that glymphatic flow deserves more attention (Aquaporin 4 and glymphatic flow have central roles in brain fluid homeostasis. Nat Rev. Neurosci. https:// doi.org/10.1038/s41583-021-00514-z $(2021))^{2}$. This topic has been reviewed extensively by researchers both in favour of $^{3}$ and in opposition to ${ }^{4,5}$ the concept (see the Review ${ }^{1}$ and correspondence for additional references). The concept was considered outside the scope of the present Review as it relates to paracellular fluid transport, as pointed out by the authors of the correspondence, rather than transmembrane water flow, which is the focus of my article (as specified in the opening pages of the Review). I therefore, again, refer the interested reader to the many reviews published on the topic. However, here, I briefly address the proposed role of AQP4 in this system in a reply to Salman and colleagues.

AQP4 has been implicated in glymphatic flow, as mentioned in the Review and highlighted by the authors of the correspondence.
Although AQP4 has been assigned a role in the paracellular flow of fluorescent probes by their reduced penetration from cerebrospinal fluid into Aqp $4^{-1-}$, Snat $1^{-1-}$ and $m d x$ mouse brains (see the correspondence for references), the mechanism by which cellular AQP4 could contribute to paracellular fluorescent tracer movement remains incompletely, if at all, understood. In the original publication by Iliff et al. ${ }^{6}$, in which the glymphatic system was coined, the authors proposed that water would flow freely through AQP4 across the astrocytic endfoot and exit again towards the interstitium (see supplementary figure 9A and associated legend in REF. and figure 1 in the correspondence ${ }^{2}$ ). The fluorescent tracer would then "follow the resulting osmotic gradient" between the paravascular space and the interstitium and thus represent glymphatic flow ${ }^{6}$. However beautiful such a strategy may be, it is challenging to align it with basic biophysical considerations. First, pressure-dependent AQP4-mediated water entry into the astrocyte would be prevented by the resulting oppositely directed osmotic gradient. Second, the proposed interstitial osmotic gradient could only arise by (the non-endfeet) AQP4 permitting water transport against an osmotic gradient, which it does not. Third, the fluorescent particles will not "follow the resulting osmotic gradient into the interstitium through intercellular clefts" ${ }^{36}$ as water follows particles by osmosis, not the opposite. Last, osmosis occurs across a semipermeable membrane and not through intercellular clefts.

To my knowledge, AQP4-dependent water transport through AQP4 has not been documented in the glymphatic hypothesis, which, however, does not prevent AQP4 from serving a structural role in the system. Notably, Aqp $4^{-/-}$mice display severely reduced expression of protein anchoring complexes in the astrocytic endfeet ${ }^{7}$, which may affect endfoot polarization of other astrocytic membrane proteins and thereby indirectly influence astrocyte function in a manner that could affect the paracellular flow of fluorescent particles. A specific and efficient inhibitor of AQP4 would provide the tool to reveal a requirement for AQP4-dependent transcellular water flux to support paracellular flow of fluorescent molecules. The authors rightly point out the futile search for such inhibitors. However, TGN-020 is among the most promising of its kind: it displays near-absent binding to $A q p 4^{-/-}$mouse brain tissue ${ }^{8}$, it causes reduced rodent brain oedema formation ${ }^{9}$ and it acts directly on the pore of AQP4 in an isoform-specific manner ${ }^{10}$. I highly welcome future determination of direct versus indirect roles of AQP4 in a glymphatic system, as well as in its pathological relocalization promoting oedema formation ${ }^{11}$, and anticipate the associated delineation of the underlying driving forces supporting the proposed AQP4-dependent transmembrane water flow. It will be my pleasure to include such mechanistic findings in future review articles on the molecular mechanisms of brain water transport.

\section{Nanna MacAulay iD Department of Neuroscience, Faculty of Health and Medical Sciences, University of Copenhagen Copenhagen, Denmark e-mail:macaulay@sund.ku.dk https://doi.org/10.1038/s41583-021-00515-y}

MacAulay, N. Molecular mechanisms of brain water transport. Nat. Rev. Neurosci. 22, 326-344 (2021). 2. Salman, M. M., Kitchen, P., Iliff, J. J. \& Bill, R. M. Aquaporin 4 and glymphatic flow have central roles in brain fluid homeostasis. Nat. Rev. Neurosci. https:// doi.org/10.1038/s41583-021-00514-z (2021).

3. Mestre, H., Mori, Y. \& Nedergaard, M. The brain's glymphatic system: current controversies. Trends Neurosci. 43, 458-466 (2020).

4. Smith, A. J. \& Verkman, A. S. The "glymphatic" mechanism for solute clearance in Alzheimer's disease: game changer or unproven speculation? FASEB J. 32 , 543-551 (2018).

5. Abbott, N. J., Pizzo, M. E., Preston, J. E., Janigro, D. \& Thorne, R. G. The role of brain barriers in fluid movement in the CNS: is there a 'glymphatic' system? Acta Neuropathol. 135, 387-407 (2018).

6. Iliff, J. J. et al. A paravascular pathway facilitates CSF flow through the brain parenchyma and the clearance 